\title{
Free Amino Acids in Human Milk and Associations with Maternal Anthropometry and
} Infant Growth

Larnkjær, Anni; Bruun, Signe; Pedersen, Dorthe; Zachariassen, Gitte; Barkholt, Vibeke; Agostoni, Carlo; Mølgaard, Christian; Husby, Steffen; Michaelsen, Kim F.

Published in:

Journal of Pediatric Gastroenterology and Nutrition

Link to article, DOI:

10.1097/MPG.0000000000001195

Publication date:

2016

Document Version

Peer reviewed version

Link back to DTU Orbit

Citation (APA):

Larnkjær, A., Bruun, S., Pedersen, D., Zachariassen, G., Barkholt, V., Agostoni, C., Mølgaard, C., Husby, S., \& Michaelsen, K. F. (2016). Free Amino Acids in Human Milk and Associations with Maternal Anthropometry and Infant Growth. Journal of Pediatric Gastroenterology and Nutrition, 63(3), 374-378.

https://doi.org/10.1097/MPG.0000000000001195

\section{General rights}

Copyright and moral rights for the publications made accessible in the public portal are retained by the authors and/or other copyright owners and it is a condition of accessing publications that users recognise and abide by the legal requirements associated with these rights.

- Users may download and print one copy of any publication from the public portal for the purpose of private study or research.

- You may not further distribute the material or use it for any profit-making activity or commercial gain

- You may freely distribute the URL identifying the publication in the public portal 
DOI : 10.1097/MPG.0000000000001195

\section{Free amino acids in human milk and associations with maternal anthropometry and infant growth}

Anni Larnkjær ${ }^{1}$, PhD, Signe Bruun ${ }^{2}$, MD, Dorthe Pedersen ${ }^{1}$, M.Sc., Gitte Zachariassen ${ }^{2}$, PhD, Vibeke Barkholt, PhD, Carlo Agostoni ${ }^{4}$, MD, Christian Mølgaard ${ }^{1,2}, \mathrm{PhD}$, Steffen Husby $^{2}$, MDSci, Kim F. Michaelsen ${ }^{1}$, MDSci.

${ }^{1}$ Department of Nutrition, Exercise and Sports, Faculty of Science, University of Copenhagen, Copenhagen, Denmark, ${ }^{2}$ Hans Christian Andersen Children's Hospital, Odense University Hospital, Odense, Denmark, ${ }^{3}$ Department of Systems Biology, Technical University of Denmark, ${ }^{4}$ Pediatric Clinic, IRCCS Ospedale Maggiore Policlinico, University of Milan, Italy

\section{Corresponding author:}

Anni Larnkjær

Rolighedsvej 26, DK-1958 Frederiksberg C, Denmark

Phone +45 353335485, (2493 secr), fax +45 35332469, email: ala@ nexs.ku.dk

Conflicts of Interest and Source of Funding:

The authors declare no conflicts of interest.

Funding declaration

The work was carried out as a part of the research programme "Governing Obesity" funded by the University of Copenhagen Excellence Programme for Interdisciplinary Research (www.go.ku.dk). The Odense Child Cohort is funded by the municipality of Odense, Odense University Hospital, Mental Health Service of the Region of Southern Denmark, and the subproject on breastfeeding has obtained support from the Danish Strategic Research Council and unrestricted support from Arla Food Ingredients.

Word count: 3107

Number of figures: 0, Number of tables: 4 


\section{ABSTRACT}

\section{Objectives}

Free glutamic acid has an appetite regulating effect and studies with infant formula have suggested that free amino acids (FAA), especially glutamic acid, can downregulate intake. The content of glutamic acid and glutamine is high in breast milk but varies considerably between mothers. The aim was to investigate if maternal anthropometry was associated with the content of the FAA glutamic acid or glutamine in breast milk and if there was a negative association between these FAA and current size or early infant growth in fully breastfed infants.

\section{Methods}

From a subgroup of 78 mothers, of which 50 were fully breast feeding, from the Odense Child Cohort breast milk samples were collected 4 months after birth and analyzed for FAA. Information regarding breastfeeding status and infant weight and length was also recorded.

\section{Results}

There was a large variation in the concentration of the FAAs between mothers. Glutamic acid was positively correlated with mother's pre-pregnancy weight and height $(p \leq 0.028)$, but not BMI. There was no negative correlation between the two FAA and infant weight or BMI. However, infant length at 4 months was positively associated with glutamine, $p=0.013$ ) but the correlation was attenuated when controlling for birth length $(p=0.089)$.

\section{Conclusions}

The hypothesis that a high content of glutamic acid and glutamine in breast milk could downregulate milk intake to a degree affecting early growth could not be confirmed. Maternal factors associated with the level of these FAA in milk and the potential effect on the infant should be investigated further. 
Key Words: breastfeeding; glutamic acid; glutamine.

\section{What is Known}

- Free glutamic acid added to infant formula and hydrolyzed formula which contains high levels of free amino acids reduces the intake of formula.

- Breastmilk contains high levels of free glutamic acid and glutamine with a large variation between mothers

- Maternal determinants of these FAA and if they influence growth in infants is not known.

\section{What is New}

- Mothers who were tall or had a high weight had higher content of glutamic acid in their milk.

- There was no negative association between content of glutamic acid or glutamine in breast milk and infant weight or BMI at 4 month.

- Glutamine content in breast milk was positively associated with infant length. 


\section{Introduction}

Formula fed infants have a higher growth velocity than breastfed infants, and it has been suggested that the main reason is the higher content of protein in formula which increases the secretion of growth promoting factors ${ }^{1-4}$. Reducing protein content in infant formula results in a growth pattern closer to breastfed infants ${ }^{5}$, but also the content of specific free amino acids (FAA), especially glutamic acid, has been suggested to have a down-regulating effect on appetite and growth. Studies utilizing hydrolyzed formula, which has a high content of FAA including glutamic acid, and also higher nitrogen content, have shown a lower intake of the hydrolyzed formula and a growth pattern, closer to the pattern of breastfed infants ${ }^{6,7}$. Furthermore, an intervention study with infant formula has shown that the addition of free glutamic acid results in a significant decrease in formula intake compared to ordinary formula ${ }^{8}$. There are receptors for free glutamic acid in the oral cavity and gastrointestinal tract and it has been suggested that glutamic acid serves as a satiety signal during feeding ${ }^{8}$. Glutamic acid and glutamine are important energy sources for the gastro intestinal mucosa, and especially glutamine is important for gut function and immune development ${ }^{9}$. Glutamine is synthesized from glutamate, but is conditional essential when the demand for glutamine is high ${ }^{10}$.

Glutamic acid and glutamine are the most abundant FAA in human milk, and increase considerably during the first months of lactation, which has been shown in several studies and in the systematic review by Zhang et al. ${ }^{11-15}$. The content of glutamic acid is the highest and increases about 1.5 times from early lactation until after 2 months, where the levels are about $1500 \mu \mathrm{mol} / \mathrm{L}^{15}$. The level of glutamine is very low in colostrum and increases to a level about $225 \mu \mathrm{mol} / \mathrm{L}$ after 2 months ${ }^{12,14,15}$. For both these FAAs there is a large variation between mothers ${ }^{10,12}$. In the study by Agostoni et al. the range at 3 months of age was $412-1941 \mu \mathrm{mol} / \mathrm{L}$ 
for glutamic acid and $138-1366 \mu \mathrm{mol} / \mathrm{L}$ for glutamine ${ }^{12}$. It is not known if there are maternal factors responsible for the large variations in these FAA.

The possible appetite regulating effect of FAA in the breastfed infant could play a role in the lower energy intake and slower growth in breastfed infants, compared to formula fed infants. Furthermore, the increase in glutamic acid and glutamine during the first months after birth could also play a role in the decrease of human milk intake per kg body weight, which happens during this period ${ }^{16}$.In addition, there is a considerable variation in the intake of breastmilk within exclusively breastfed infants and in the weight gain between healthy exclusively breastfed infants. Using the WHO Growth Standard curves the range in weight gain from 1-4 months of age for the $5^{\text {th }}$ and $95^{\text {th }}$ percentile are about $1.6 \mathrm{~kg}$ and $3.3 \mathrm{~kg}$ (average of boys and girls) ${ }^{17}$.

A dose-response association is an essential prerequisite to support the biologic plausibility of a direct causality of a given compound on a hypothetical effect. To examine the potential appetite regulating effect of FAA in human milk and thereby the effect on growth we measured the two most abundant FAA in human milk; glutamic acid and glutamine at the age of about 4 months in a cohort of healthy breastfed infants. The aim was to investigate if there was an association between the content of these two FAA in human milk and maternal anthropometry, as well as with current infant size or increase in weight, length and BMI since birth.

\section{METHODS}

\section{Subjects}

Subjects were a subgroup of mothers and their infants participating in the prospective birth cohort Odense Child Cohort which has been described in detail elsewhere ${ }^{18}$. Briefly, 
approximately 2500 pregnant mothers living in the municipality of Odense were included from 2010-2012 and followed through pregnancy and early childhood. Included infants were born at term. The children were examined at 4 months of age, including measurement of weight to the nearest gram and recumbent length in centimeter with one decimal on a digital baby scale with a built in measuring rod (Seca 717, Hamburg, Germany). The mothers were given the opportunity to deliver a breast milk sample when attending the examination at about 4 months of age. There was no requirement for how the breast milk sample was taken except from a desired amount of $30 \mathrm{~mL}$. It was not recorded if it was fore- or hind-milk. Full breastfeeding included infants who never had any formula or only formula up to 1 week after birth until the breastfeeding was fully established; otherwise they were considered partially breastfed. For this study a random selection of 78 samples was used. Birth data and maternal height and pre-pregnancy weight were obtained from birth records and questionnaires, respectively.

The Regional Scientific Ethical Committee for Southern Denmark approved the study, (S20090130).

\section{Analysis of FAA and macronutrients}

Initially, expressed breast milk (approx. $30 \mathrm{~mL}$ ) was mixed, centrifuged at $2000 \mathrm{~g}$ for 5 minutes and separated into an upper layer (fat phase) and an intermediate layer and the pellet. The intermediate phase was frozen at $-20^{\circ} \mathrm{C}$ and shipped frozen for further processing. The FAA were analyzed at the BioCentrum at the Danish Technical University. For the FAA analysis the samples were thawed, mixed with the internal standard Norleucin $(0.5 \mathrm{nmol} / \mu \mathrm{l})$ and centrifuged at $3000 \mathrm{~g}$ for $5 \mathrm{~min}$. The fatty phase was discarded and proteins were precipitated with $6 \%$ 
sulfosalicylic acid. After $10 \mathrm{~min}$ storage in the freezer and centrifugation for $10 \mathrm{~min}$ at $20.000 \mathrm{~g}$ the supernatant was removed and frozen until analysis. The FAA were analyzed by reversedphase high performance liquid chromatography (HPLC) with ultraviolet and fluorescence detector using a AccQ-Tag column (3.9 x $150 \mathrm{~mm})$ (Waters Division, Milford, MA, US) and pre-column derivatization with AccQ-Flour Reagent Kit (Waters Division, Milford, MA, US) ${ }^{19}$. Amino acids derivatives were separated in a ternary gradient consisting of solvent A: $140 \mathrm{mM}$ sodium acetate, $7.0 \mathrm{mM}$ triethylamine, $1.0 \mathrm{mg} / \mathrm{L}$ ethylenediaminetetraacetic acid, $\mathrm{pH}=5.7$; solvent B: $60 \%$ acetonitrile and eluent C: water with a run time of $66 \mathrm{~min}$. The samples were analyzed in duplicate.

Fat, protein and lactose were measured by mid-infrared transmission spectroscopy using a Human Milk Analyzer from Miris AB, Uppsala, Sweden. For these analyses frozen samples of whole human milk were defrosted in a refrigerator, heated in warm water until reaching a temperature of $40{ }^{\circ} \mathrm{C}$ and homogenized before analysis as described previously ${ }^{20}$.

\section{Statistical analysis}

Z-scores for weight, length, weight-for-length and BMI were calculated using the software program WHO Anthro 2007 (Department of Nutrition, WHO, Geneva, Switzerland). Data were analyzed using IBM SPSS statistics (version 21, IBM, NY, US). Significance was defined as $p$ values $<0.05$ and trends as $p$-values $<0.10$. Descriptive statistics are given by mean \pm SD and range and for the FAA also 10, 25, 50, 75 and 90 percentiles. Differences in anthropometry, maternal characteristics or FAA between fully breastfed and partially breastfed infants were analyzed by independent $t$ test, Mann-Whitney or chi-squared test as appropriate. The bivariate correlations between the FAA and infants size at birth or maternal characteristics were conducted 
by Pearson correlation or Spearman's rho as appropriate using all subjects. The same procedure was applied for the correlations between the FAA and the macronutrients. Correlations adjusted for infant age were conducted using partial correlation. The associations between the FAA and infant anthropometry at the examination were investigated by multiple linear regression using general linear models. Residual plots and Cooks distance were used for model verification. We evaluated the associations in different models. The crude model only included adjustment for age and gender. The adjusted models included baseline values (values on weight, length and BMI at birth) i.e. the beta-values correspond to the change from birth to the examination at 4 month. Furthermore, adjusted models also included breastfeeding status (fully or partially) and maternal values (mothers pre-pregnancy BMI for infant weight and BMI as dependent variable, and mothers' height for infant length as dependent variable). The same models were performed only on infants fully breastfed. As inclusion of only fully breastfed infant did not change the significance and the results were similar for the adjusted models only the results for the model adjusted for baseline value, gender and age including only fully breastfed infants are presented.

\section{RESULTS}

The characteristics of the subjects are shown in Table 1. The sample consisted of 78 children (49 $\%$ girls). Of these, 50 and 21 infants were fully or partially breastfed, respectively, and breastfeeding status was unknown for seven infants. Fifty infants were classified as fully breastfed. Ten of these received formula for only the first week while the remaining infants were exclusively breastfed. Among the 21 partially breastfed infants seven were getting formula at the 4 month visit and of these four also received complementary foods. Among the remaining 14 partially breastfed infants six had started on complementary foods at the 4 month visit and the 
remaining had received formula for mainly short periods after the age of one week but not when they were 4 months. There was no difference in the concentration of FAA in breast milk between mothers, who were fully or partially breastfeeding (data not shown). As regards the anthropometric measures of the infants only the weight at the examination differed (mean weight \pm SD fully breast fed: $7276 \pm 767 \mathrm{~g}$, partially breastfed: $7714 \pm 771 \mathrm{~g} ; p=0.032$ ) which is due to the higher age of the partially breastfed infants (mean age \pm SD fully breastfed: $18.4 \pm 1.9 \mathrm{wk}$, partially breastfed: $20.0 \pm 2.7 \mathrm{wk} ; p=0.019)$. In accordance with this there was no difference in weight for age $\mathrm{z}$-score (WAZ) between partially and fully breastfed infants ( $p=0.127)$.

The concentrations of the FAA glutamic acid and glutamine are shown in Table 2. Glutamic acid was the most abundant FAA, and both amino acids showed a broad concentration range. The level of the two FAA did not correlate with each other $(\mathrm{p}=0.592)$. There was no significant associations between the infant's age and the concentration of FAA (glutamic acid r=-0.139, $p=0.225$; glutamine $\mathrm{r}=-0.092, p=0.425$ ). The total content of the $21 \mathrm{FAA}$ we analyzed was 38.68 $\pm 5.07 \mu \mathrm{g} / 100 \mu \mathrm{L}($ mean $\pm \mathrm{SD})$. Glutamic acid and glutamate constituted $38 \pm 5 \%(\mathrm{w} / \mathrm{w})$ and $10 \pm$ $3 \%(\mathrm{w} / \mathrm{w})$ of this, respectively. Data on macronutrients were only available for 27 subjects and there were no significant correlations between the content of FAA and the content of any of the macronutrients $(p \geq 0.349$; data not shown).

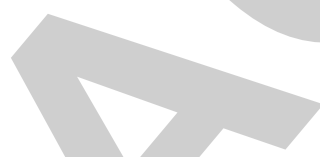

Glutamic acid was positively associated with mothers pre-pregnancy weight and height $(\mathrm{r}=0.264$, $p=0.020$ and $\mathrm{r}=0.249, p=0.028$, respectively). Controlling for the infants ${ }^{\prime}$ age strengthened these associations (Table 3). There were no significant associations with the mothers pre-pregnancy 
BMI. However, glutamine tended to be negatively associated with pre-pregnancy BMI ( $\mathrm{r}=-0.212$, $p=0.063$ ) but the association was weakened by adjustment for infants' age.

Glutamine was positively correlated with birth length $(\mathrm{r}=255, p=0.024)$ but there was no association with birth weight. Glutamic acid was not associated with birth weight or length .

The associations between FAA in the milk samples and current infant size examined by multiple regression analyses are presented in Table 4 for fully breastfed infants.. Current length was positively associated with glutamine, but adjustment for birth length attenuated the association. For BMI there was no significant association with the FAA. Adjustment for maternal values had no significant effect on the associations (data not shown).

Analyses on all subjects $(\mathrm{n}=78)$ showed a trend for a positive association between the concentration of glutamic acid and current weight adjusted for gender and age ( $p=0.076$, Crude Model; data not shown). When birth weight was included in the model (Adjusted Model) the trend disappeared. Otherwise there was no difference compared to the analyses performed on only fully breastfed infants and inclusion of breastfeeding status in the adjusted model did not change the results.

\section{DISCUSSION}

The hypothesis that the content of free glutamic acid in human milk has an appetite regulating effect influencing term born infant weight gain, so infants receiving human milk with a high content of free glutamic acid would have a lower weight gain, could not be confirmed in this 
study. However, based on this study we cannot exclude that glutamic acid in breastmilk has an appetite regulating effect as we measured infant growth as a crude indicator of milk intake. We found a positive association between free glutamine content and infant length but this was only a trend when adjusted for birth length (corresponding to growth in length from birth to 4 month). Birth length was positively associated with the glutamine content in the milk at 4 months. We have no explanation why mothers with a high content of glutamine in their milk deliver infants born with a high length, except speculating on possible endocrine-mediated mechanisms where low levels of glutamine and/or glutamic acid are possible limiting factors ${ }^{21}$ even if the biologic plausibility mostly stands for a role of glutamic acid in synaptic mechanisms 22. A finding in the same direction was seen in a study comparing glutamine in milk at one month between small for gestational age (SGA) and appropriate for gestational age (AGA) infants, showing higher levels in AGA infants ${ }^{23}$. There was no information on birth length. However, the studies are difficult to compare due to difference in stage of lactation and lack of SGA infants in our study. In our study we found no associations between birth weight and the FAA at 4 month. These findings could highlight more complex mechanisms in the maternalinfant dyads, not only limited to lactation.

To confirm the possible role of a biologic regulation linking the mother-infant dyad through glutamic acid, we found that mothers with a high weight and stature had a significant higher content of free glutamic acid in their milk. For BMI it was only a trend $(\mathrm{p}<0.099)$. The association with weight was quite strong $(\mathrm{p}=0.005)$ suggesting that it was not a spurious finding. An increase of $1 \mathrm{~kg}$ of the mother's weight corresponded to an increase of $5.6 \mu \mathrm{mol} / \mathrm{L}$ in glutamic acid concentration. To our knowledge this has not been reported before. If free 
glutamate has a down regulating effect on appetite and thereby milk intake, it is interesting that large mothers have high levels of free glutamic acid in their milk. Associations between free glutamate in human milk and maternal anthropometry have been examined in a study by Jochum et al. They reported maternal BMI and weight in the first trimester to be negatively associated with free glutamine in early breast milk (days 4-7 of lactation) ${ }^{10}$ and in accordance with this we found a trend $(\mathrm{p}=0.09)$ for an inverse correlation between pre-pregnancy BMI and free glutamine in mature milk. We have no suggestions for mechanisms for how maternal anthropometry is associated with the content of FAA in their breast milk, but since these FAA have important physiologic effects it would be interesting to study this further. Again, unknown endocrine and receptor mediated mechanisms may offer a biologically plausible explanation, but this should be explored in more detail.

Whether the infant was fully or partially breastfed had no influence on the relation between the FAA and size of the infant. A possible reason for this might be that only seven of the partially breastfed infants were receiving formula at the 4 months visit. Therefore there might not be a large difference between partially and fully breastfed infants regarding intake of FAA in this study.

There was a large variation in the content of amino acids between mothers as described in the literature ${ }^{12,15,23,24}$. The glutamic acid and glutamine concentrations in this study were comparable to those reported at 3 months ${ }^{12}$ and $\geq 2$ months ${ }^{15}$. According to Zhang ${ }^{15}$ the content of glutamine and glutamic acid increases after birth and peaks between 3 and 6 months. We did not find an increase in glutamine and glutamic acid with progression of the lactation stage as most samples were obtained at 4 months or within a narrow time range around 4 months, when 
the concentration of glutamine and glutamic acid are not expected to change considerably ${ }^{15,24}$. Also the methods applied for determination of FAA may affect the measurements and newer methods may have improved the quantification. Often HPLC methods or automatic amino acid analyzers are used for quantification ${ }^{10,14,15,23-25}$. The reverse-phase HPLC method we have applied, resulted in levels of FAA in accordance with levels reported in other studies. We have also referred to a paper by WU et al. ${ }^{13}$ using nuclear magnetic resonance (NMR) spectroscopy for FAA quantification. The levels of glutamic acid and glutamate in mature milk are higher compared to values reported in this paper and the review ${ }^{15}$ but as the NMR values are only based on samples from one female it is difficult to conclude anything regarding the NMR method.

The study has several limitations. The sample size was relatively small so the possibility of chance finding cannot be excluded. Furthermore, we used growth from birth to 4 months as a surrogate for average milk intake during this period. The strength of the study was the large variation in FAA and in weight, length and BMI making it possible to investigate the associations over a wide range.

Other factors which might influence growth are the appetite related hormones in human milk, such as leptin, adiponectin and ghrelin ${ }^{26-29}$ which were not measured. These hormones may play a role in regulating the appetite despite the immaturity of the appetite control system in infancy 26,30. High levels of appetite regulating hormones in breast milk have been associated with high weight gain in early infancy ${ }^{31}$. Thus, the appetite regulation may be different in formula fed infants regarding the effect of ingested appetite regulatory hormones as the presence of these hormones in formula is uncertain ${ }^{29,32,33}$. Another shortcoming of the study is the observational 
design so only associations can be shown and no causative conclusions can be made. Studies to support the findings and elucidate possible mechanisms are therefore warranted.

To summarize in this cohort of breastfed infants the content of the FAA glutamic acid and glutamine does not seem to be related to weight gain or adiposity measured by BMI but glutamine may be positively related to growth in length. Furthermore, glutamic acid was positively associated with maternal weight and height. The cause and potential effects of these associations need to be explored further, as well as the possible mechanisms, since both these amino acids have important functions in the infant.

\section{Acknowledgements}

The authors gratefully acknowledge Vivian Anker and Mette Vogn Hviid for technical assistance. Anne Blicher, the late Susanne Jacobsen both from Systems Biology, Technical University of Denmark performed the amino acids analyses and helped with describing the methods, which we gratefully acknowledge. 


\section{References}

1. Dewey KG, Heinig MJ, Nommsen LA, et al. Growth of breast-fed and formula-fed infants from 0 to 18 months: the DARLING Study. Pediatrics 1992;89:1035-41.

2. Roche AF, Guo S, Siervogel RM, et al. Growth comparison of breast-fed and formula-fed infants. Can J Public Health 1993;84:132-5.

3. Madsen AL, Larnkjaer A, Molgaard C, et al. IGF-I and IGFBP-3 in healthy 9month old infants from the SKOT cohort: Breastfeeding, diet, and later obesity. Growth Horm IGF Res 2011;4:199-204.

4. Madsen AL, Schack-Nielsen L, Larnkjaer A, et al. Determinants of blood glucose and insulin in healthy 9-month-old term Danish infants; the SKOT cohort. Diabet Med 2010;12:1350-7.

5. Koletzko B, von KR, Closa R, et al. Lower protein in infant formula is associated with lower weight up to age 2 y: a randomized clinical trial. Am J Clin Nutr 2009;89:1836-45.

6. Mennella JA, Ventura AK, Beauchamp GK. Differential growth patterns among healthy infants fed protein hydrolysate or cow-milk formulas. Pediatrics 2011;127:110-8.

7. Rzehak P, Sausenthaler S, Koletzko S, et al. Short- and long-term effects of feeding hydrolyzed protein infant formulas on growth at $<$ or $=6 \mathrm{y}$ of age: results from the German Infant Nutritional Intervention Study. Am J Clin Nutr 2009;89:1846-56.

8. Ventura AK, Beauchamp GK, Mennella JA. Infant regulation of intake: the effect of free glutamate content in infant formulas. Am J Clin Nutr 2012;95:875-81.

9. Jacobi SK and Odle J. Nutritional factors influencing intestinal health of the neonate. Adv Nutr 2012;5:687-696.

10. Jochum F, Colling S, Meinardus $\mathrm{P}$, et al. Total glutamine content in human milk is not influenced by gestational age. Acta Paediatr 2006;8:985-90. 
11. Atkinson SA and Lonnerdal B. Nonprotein nitrogen fractions of human milk. In: Jensen RG, ed. Handbook of Milk Composition, San Diego: Academic Press; 1995;369-87.

12. Agostoni C, Carratu B, Boniglia C, et al. Free glutamine and glutamic acid increase in human milk through a three-month lactation period. J Pediatr Gastroenterol Nutr 2000;31:50812.

13. Wu J, Domellof M, Zivkovic AM, et al. NMR-based metabolite profiling of human milk: A pilot study of methods for investigating compositional changes during lactation. Biochem Biophys Res Commun 2016;469:626-32.

14. Baldeon ME, Mennella JA, Flores N, et al. Free amino acid content in breast milk of adolescent and adult mothers in Ecuador. Springerplus 2014;3:104-7.

15. Zhang Z, Adelman AS, Rai D, et al. Amino acid profiles in term and preterm human milk through lactation: a systematic review. Nutrients 2013;5:4800-21.

16. Neville MC, Keller R, Seacat J, et al. Studies in human lactation: milk volumes in lactating women during the onset of lactation and full lactation. Am J Clin Nutr 1988;6:1375-86.

17. Wold Health Organization. The WHO Child Growth Standards.

http://www.who.int/childgrowth/en/. Accessed January 22, 2016.

18. Kyhl HB, Jensen TK, Barington T, et al. The Odense Child Cohort: aims, design, and cohort profile. Paediatr Perinat Epidemiol 2015;29:250-8.

19. Wandelen CV and Cohen S. Using quaternary high-performance liquid chromatography eluent systems for separating 6-aminoquinolyl-N-hydroxysuccinimidyl carbamate-derivatized amino acid mixtures. J Chromatogr 1997;763:11-22.

20. Zachariassen G, Fenger-Gron J, Hviid MV, et al. The content of macronutrients in milk from mothers of very preterm infants is highly variable. Dan Med J 2013;60:A4631 
21. Parent AS, Lebrethon MC, Gerard A, et al. Factors accounting for perinatal occurrence of pulsatile gonadotropin-releasing hormone secretion in vitro in rats. Biol Reprod 2005;72:143-9. 22. Tani H, Dulla CG, Farzampour Z, et al. A local glutamate-glutamine cycle sustains synaptic excitatory transmitter release. Neuron 2014;81:888-900.

23. Pamblanco M, Portoles M, Paredes C, et al. Free amino acids in preterm and term milk from mothers delivering appropriate- or small-for-gestational-age infants. Am J Clin Nutr 1989;50:778-81.

24. Yamawaki N, Yamada M, Kan-no T, et al. Macronutrient, mineral and trace element composition of breast milk from Japanese women. J Trace Elem Med Biol 2005;19:171-181.

25. Agostoni C, Carratu B, Boniglia C, et al. Free amino acid content in standard infant formulas: comparison with human milk. J Am Coll Nutr 2000;4:434-8.

26. Savino F, Fissore MF, Liguori SA, et al. Can hormones contained in mothers' milk account for the beneficial effect of breast-feeding on obesity in children? Clin Endocrinol (Oxf) 2009;71:757-65.

27. Catli G, Olgac DN, Dundar BN. Adipokines in breast milk: an update.. J Clin Res Pediatr Endocrinol 2014;6:192-201.

28. Bielicki J, Huch R, von MU. Time-course of leptin levels in term and preterm human milk. Eur J Endocrinol 2004;151:271-6.

29. Savino F, Liguori SA. Update on breast milk hormones: leptin, ghrelin and adiponectin. Clin Nutr 2008;27:42-7.

30. Hassiotou F, Geddes DT. Programming of appetite control during breastfeeding as a preventative strategy against the obesity epidemic. J Hum Lact 2014;30:136-42. 
31. Kon IY, Shilina NM, Gmoshinskaya MV, et al. The study of breast milk IGF-1, leptin, ghrelin and adiponectin levels as possible reasons of high weight gain in breast-fed infants. Ann Nutr Metab 2014;65:317-23.

32. Resto M, O'Connor D, Leef K, et al. Leptin levels in preterm human breast milk and infant formula. Pediatrics 2001;108:E15

33. O'Connor D, Funanage V, Locke R, et al. Leptin is not present in infant formulas. J Endocrinol Invest 2003;26:490. 
Table 1. Characteristics of infants and mothers

\begin{tabular}{|c|c|c|c|c|}
\hline & Mean & SD & Range & Number $(\%)$ \\
\hline \multicolumn{5}{|l|}{ Infants at Birth } \\
\hline Gender: Girls/Boys & & & & $38(49) / 40(51)$ \\
\hline Weight (g) & 3615 & 494 & $2495-4870$ & \\
\hline Length $(\mathrm{cm})$ & 52.6 & 2.0 & $49-59$ & \\
\hline \multicolumn{5}{|l|}{ Infants at Examination } \\
\hline Age (months) & 4.31 & 0.54 & $3.20-6.07$ & \\
\hline Weight (g) & 7280 & 895 & $5120-9260$ & \\
\hline Length (cm) & 65.0 & 2.3 & $59.5-71.0$ & \\
\hline BMI $\left(\mathrm{kg} / \mathrm{m}^{2}\right)$ & 17.2 & 1.8 & $13.4-22.3$ & \\
\hline Weight z-score & 0.44 & 0.97 & $-2.78-2.53$ & \\
\hline Length z-score & 0.64 & 0.97 & $-2.22-3.01$ & \\
\hline Weight-for-length z-score & 0.11 & 1.18 & $-2.51-3,01$ & \\
\hline BMI-z-score & 0.11 & 1.16 & $-2.32-2.98$ & \\
\hline \multicolumn{5}{|l|}{ Breastfeeding status } \\
\hline Exclusively/partially/n.a. & & & & $50(64) / 21(27) / 7(9)$ \\
\hline \multicolumn{5}{|l|}{ Maternal characteristics } \\
\hline Pre-pregnancy weight (kg) & 69.7 & 13.5 & $48.0-116.0$ & \\
\hline Height $(\mathrm{cm})$ & 170.0 & 6.9 & $156-189$ & \\
\hline Pre-pregnancy BMI $\left(\mathrm{kg} / \mathrm{m}^{2}\right)$ & 24.1 & 4.7 & $18.1-42.6$ & \\
\hline
\end{tabular}

BMI body mass index, z-score Standard deviation score 
Table 2. Content of free amino acids ${ }^{a}$

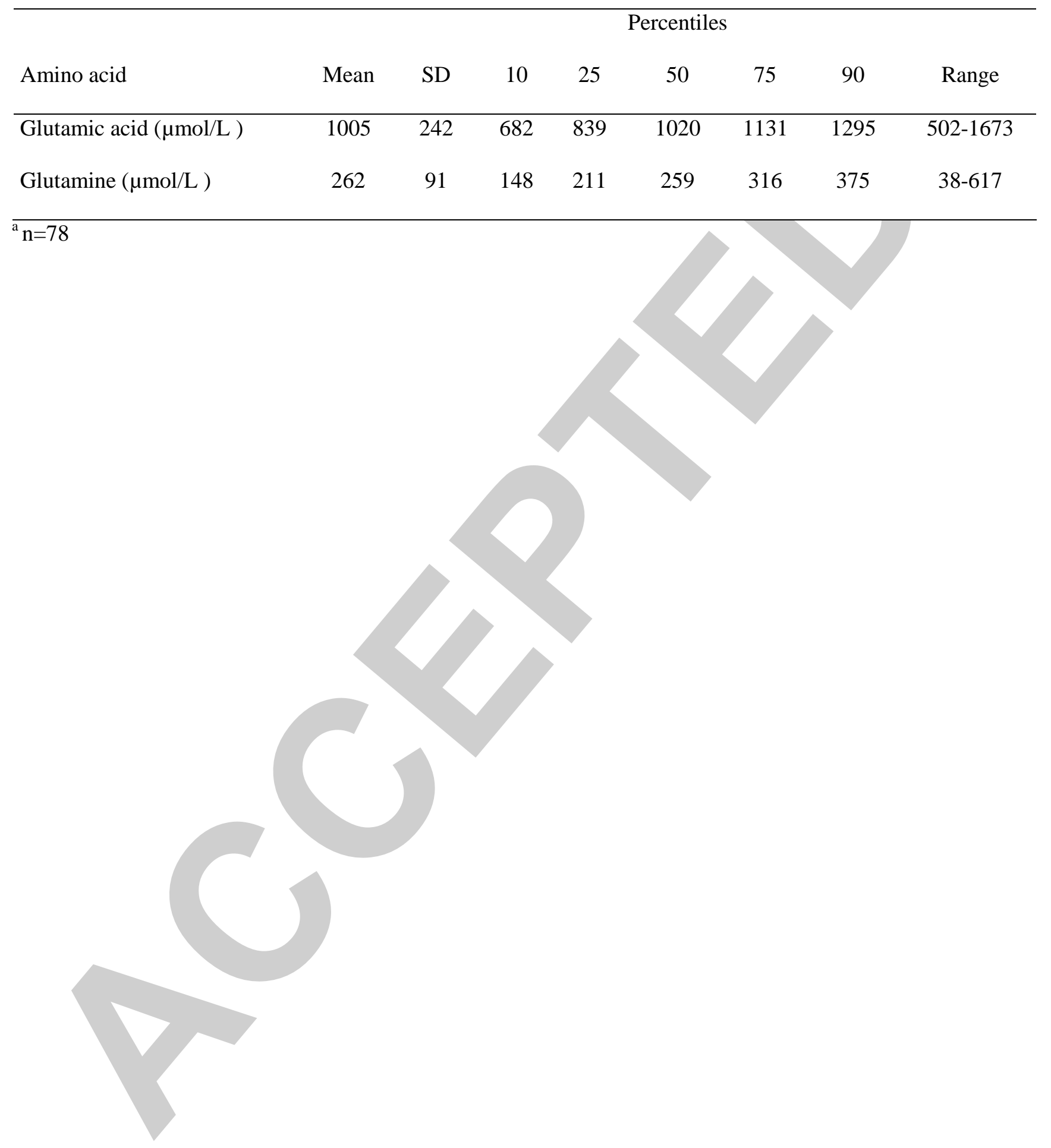


Table 3. Associations between free amino acids in breastmilk and size of the mothers adjusted for the infants age ${ }^{\mathrm{a}}$.

\begin{tabular}{lccc}
\hline Amino Acid & Pre-pregnancy weight $(\mathrm{kg})$ & Height $(\mathrm{cm})$ & Pre-pregnancy BMI $\left(\mathrm{kg} / \mathrm{m}^{2}\right)$ \\
\hline Glutamic acid $(\mu \mathrm{mol} / \mathrm{L})$ & $0.314(0.005)$ & $0.282(0.013)$ & $0.189(0.099)$ \\
Glutamine $(\mu \mathrm{mol} / \mathrm{L})$ & $-0.178(0.122)$ & $0.053(0.649)$ & $-0.194(0.092)$
\end{tabular}

${ }^{\mathrm{a}}$ Values are $\mathrm{r}(p), \mathrm{n}=78$. BMI body mass index. 
Table 4. Associations between the free amino acids and infants size at the examination for infants fully breastfed.

\begin{tabular}{|c|c|c|c|c|}
\hline \multirow[t]{2}{*}{ Parameters } & \multicolumn{2}{|c|}{ Crude Model ${ }^{\mathrm{a}}$} & \multicolumn{2}{|c|}{ Adjusted Model $^{\mathrm{b}}$} \\
\hline & $\beta[\mathrm{CI}]$ & $p$ value & & $p$ value \\
\hline \multicolumn{5}{|l|}{ Weight $(g)$} \\
\hline Glutamic acid $(\mu \mathrm{mol} / \mathrm{L})$ & $0.575[-0.223,1.373]$ & 0.154 & $0.494[-0.289,1.277]$ & 0.211 \\
\hline Glutamine $(\mu \mathrm{mol} / \mathrm{L})$ & $0.423[-1.795,2.641]$ & 0.70 & $0.210[-1.955,2.376]$ & 0.846 \\
\hline \multicolumn{5}{|l|}{ Length $(\mathrm{cm})$} \\
\hline Glutamic acid $(\mu \mathrm{mol} / \mathrm{L})$ & $0.002[-0.001,0.004]$ & 0.143 & $0.001[0.000,0.003]$ & 0.106 \\
\hline Glutamine $(\mu \mathrm{mol} / \mathrm{L})$ & $0.007[0.002,0.013]$ & 0.013 & $0.004[-0.001,0.009]$ & 0.089 \\
\hline \multicolumn{5}{|l|}{$B M I\left(k g / m^{2}\right)$} \\
\hline Glutamic acid $(\mu \mathrm{mol} / \mathrm{L})$ & $0.000[-0.001,0.002]$ & 0.596 & $0.000[-0.001,0.002]$ & 0.665 \\
\hline Glutamine $(\mu \mathrm{mol} / \mathrm{L})$ & $-0.003[-0.008,0.002]$ & 0.231 & $-0.003[-0.008,0.002]$ & 0.248 \\
\hline
\end{tabular}

${ }^{\mathrm{a}}$ Crude model included control for age and gender, $\mathrm{n}=50$

${ }^{\mathrm{b}}$ Adjusted Model included control for age, gender and value at birth, $\mathrm{n}=50$

BMI body mass index 